\title{
Influence of an algal triacylglycerol containing docosahexaenoic acid $(22: 6 n-3)$ and docosapentaenoic acid $(22: 5 n-6)$ on cardiovascular risk factors in healthy men and women
}

\author{
Thomas A.B. Sanders ${ }^{1}$, Kelly Gleason ${ }^{1}$, Bruce Griffin ${ }^{2}$ and George J. Miller ${ }^{3}$ \\ ${ }^{1}$ Nutritional Sciences Research Division, Franklin-Wilkins Building, King's College London, 150 Stamford Street, London SE1 9NH, UK \\ ${ }^{2}$ Centre for Nutrition \& Food Safety, School of Biomedical \& Life Sciences, University of Surrey, Guildford, Surrey, UK \\ ${ }^{3}$ Wolfson Institute, Royal London \& St Bartholomew's Hospital Medical School, London, UK
}

(Received 27 April 2005 - Revised 11 October 2005 - Accepted 29 October 2005)

\begin{abstract}
The intake of long-chain $n$-3 PUFA, including DHA (22:6n-3), is associated with a reduced risk of CVD. Schizochytrium sp. are an important primary source of DHA in the marine food chain but they also provide substantial quantities of the $n$ - 6 PUFA docosapentaenoic acid (22:5n-6; DPA). The effect of this oil on cardiovascular risk factors was evaluated using a double-blind randomised placebo-controlled parallel-design trial in thirty-nine men and forty women. Subjects received $4 \mathrm{~g}$ oil $/ \mathrm{d}$ for 4 weeks; the active treatment provided $1.5 \mathrm{~g}$ DHA and $0.6 \mathrm{~g}$ DPA. Active treatment increased plasma concentrations of arachidonic acid, adrenic acid, DPA and DHA by $21,11,11$ and $88 \mathrm{mg} / 1$ respectively and the proportions of DPA and DHA in erythrocyte phospholipids by 78 and $27 \%$ respectively. Serum total, LDL- and HDL-cholesterol increased by $0.33 \mathrm{mmol} / \mathrm{l}(7.3 \%), 0.26 \mathrm{mmol} / 1(10 \cdot 4 \%)$ and $0.14 \mathrm{mmol} / 1(9.0 \%) \mathrm{com}-$ pared with placebo (all $P \leq 0.001$ ). Factor VII (FVII) coagulant activity increased by $12 \%$ following active treatment $(P=0.006)$. There were no significant differences between treatments in LDL size, blood pressure, plasma glucose, serum C-reactive protein, plasma FVII antigen, FVII activated, fibrinogen, von Willebrand factor, tocopherol or carotenoid concentrations, plasminogen activator inhibitor-1, creatine kinase or troponin-I activities, haematology or liver function tests or self-reported adverse effects. Overall, the oil was well tolerated and did not adversely affect cardiovascular risk.
\end{abstract}

Docosahexaenoic acid: Docosapentaenoic acid: Lipids: Haemostasis: C-reactive protein

Prospective cohort studies indicate that the intake of EPA $(20$ : $5 n-3)$ and DHA $(22: 6 n-3)$ derived from the consumption of oily fish is associated with a lower risk of fatal IHD (Albert et al. 1998; Hu et al. 2002) and stroke (Mozaffarian et al. 2005). A meta-analysis of secondary prevention trials of IHD concluded that an intake in the range of $0.7-1 \mathrm{~g} / \mathrm{d}$ of a mixture of EPA and DHA decreased the incidence of sudden cardiac death (Bucher et al. 2002). This beneficial effect appeared to occur rapidly following dietary intervention (Marchioli et al. 2002), implying that it was mediated by a process that was amenable to short-term influences such as a decreased susceptibility of an atherosclerotic plaque to rupture or an amelioration of the consequences of plaque rupture such as thrombosis or ventricular fibrillation. It is currently uncertain whether EPA or DHA or both together are responsible for this protective effect. Besides elevated blood pressure and dyslipidaemia, several pro-inflammatory and haemostatic factors are known to predict the risk of cardiovascular events such as elevated C-reactive protein, fibrinogen and von Willebrand factor concentrations, and plasminogen activator type 1 (PAI-1) and factor VII coagulant (FVIIc) activities (Meade et al. 1993; Thompson et al. 1995; Cooper et al. 2000; Ridker et al. 2000). However, the effects of DHA in isolation from EPA on these risk factors are uncertain.
DHA can be synthesised from linolenic acid $(18: 3 n-3)$ in mammalian tissues but its formation is limited by feedback inhibition and by competition from linoleic acid (18:2n-6) (Makrides et al. 2000; Rosell et al. 2005). Preformed DHA appears to be a major determinant of the proportion of DHA in membrane lipids. The richest dietary source of DHA is oily fish, but small amounts are also provided by eggs and meat (British Nutrition Foundation, 1992). The high proportions of EPA and DHA in oily fish result from the accumulation of lipids synthesised by marine algae. Unlike DHA from land animals which is derived by synthesis from linolenic acid, DHA in algae has been shown to be derived via a novel metabolic pathway that does not involve linolenic acid as substrate (Metz et al. 2001). Although DHA from Crypthecodinium cohnii is widely used in the formulation of breast-milk substitutes, there have been few thorough investigations of DHA from algal sources on cardiovascular risk factors. Theobald et al. (2004) reported a daily intake of $0.7 \mathrm{~g}$ DHA provided as a triacylglycerol derived from $C$. cohnii taken for 3 months resulted in a $7 \%$ increase in LDL-cholesterol. Oil from Schizochytrium $s p$. is also rich in DHA, but unlike that derived from C. cohnii, it contains about $15 \%$ docosapentaenoic acid $(22: 5 n-6 ;$ DPA). The aim of the present study was to undertake a comprehensive evaluation of a purified

Abbreviations: CRP, C-reactive protein; DHA-S, DHA-rich triacylglycerol derived from Schizochytrium sp., DPA, docosapentaenoic acid; FVIIa, factor VII activated; FVIIag, factor VII antigen; FVIIc, factor VII coagulant; PAI-1, plasminogen activator inhibitor-1; TAG, triacylglycerol.

* Corresponding author: Professor T. A. B Sanders, fax +44 207848 4171, email Tom.Sanders@kcl.ac.uk 
triacylglycerol (TAG) derived from Schizochytrium sp. on cardiovascular risk factors in healthy men and women.

\section{Subjects, materials and methods}

\section{Design}

A randomised double-blind placebo-controlled parallel design was selected because of the long carry-over effect of DHA in membrane lipids. Equal numbers of males and females were allocated to each treatment by stratified randomisation, which was conducted by an independent company (Rho Inc., Chapel Hill, NC, USA). Treatment allocation was withheld from the investigators until the data analysis was complete (except for the post hoc analyses). Subjects were asked to consume four gelatin capsules daily; each capsule contained $1 \mathrm{~g}$ oil. Both active and placebo capsules were manufactured to contain similar amounts of antioxidants and vitamin E. The active treatment consisted of $4 \mathrm{~g}$ refined DHA-rich TAG derived from Schizochytrium $s p$. (DHA-S) and the placebo consisted of $4 \mathrm{~g}$ refined olive oil. The fatty acid composition of the supplements is shown in Table 1 . Both treatments provided $148 \mathrm{~kJ}$ and $4 \mathrm{~g}$ fat. The active treatment provided $1.5 \mathrm{~g}$ DHA, $0.6 \mathrm{~g}$ DPA, $0.04 \mathrm{~g}$ MUFA and $1.2 \mathrm{~g}$ saturated fatty acids (mainly myristic and palmitic acid). The placebo provided $0.6 \mathrm{~g}$ PUFA (mainly linoleic acid), $2.7 \mathrm{~g}$ MUFA (mainly oleic acid) and $0.5 \mathrm{~g}$ saturated fatty acids (mainly palmitic acid). Responses were assessed by measurements made on two occasions at baseline and on days 28 and 29 of each treatment.

\section{Subjects}

Subjects were recruited by a circular email from within the staff population of King's College London, St Thomas', Guy's and King's College Hospitals, all located in London. Exclusion criteria were: current tobacco use; history of myocardial infarction or diabetes mellitus; current pregnancy; current use of lipid-lowering or blood pressure-lowering or immunosuppressive drugs; hormone replacement therapy; use of systemic corticosteroids, androgens, phenytoin or erythromycin, thyroid hormone, drugs for regulating haemostasis but excluding aspirin; self-reported alcohol intake $>21$ units/week for women and $>28$ units/week for men (1 unit $=10 \mathrm{ml}$ ethanol); BMI $<18$ or $>35 \mathrm{~kg} / \mathrm{m}^{2}$; serum cholesterol $>7.8 \mathrm{mmol} / \mathrm{l}$ or fasting serum TAG $>3.0 \mathrm{mmol} / \mathrm{l}$; systolic blood pressure $>140 \mathrm{mmHg}$ or diastolic blood pressure $>90 \mathrm{mmHg}$; abnormal haematology or liver function test. Subjects were also

Table 1. Fatty acid content $(\mathrm{mg} / \mathrm{g})$ of docosahexaenoic acid-rich oil derived from Schizochytrium sp. (DHA-S) and placebo

\begin{tabular}{lrc}
\hline Fatty acid & DHA-S & Placebo \\
\hline $14: 0$ & $75 \cdot 1$ & 0 \\
$16: 0$ & $203 \cdot 9$ & $124 \cdot 0$ \\
$16: 1$ & $4 \cdot 1$ & $13 \cdot 7$ \\
$18: 0$ & $5 \cdot 1$ & $24 \cdot 7$ \\
$18: 1$ & $5 \cdot 6$ & $652 \cdot 9$ \\
$18: 2 n-6$ & $4 \cdot 5$ & $119 \cdot 8$ \\
$20: 4 n-6$ & $7 \cdot 3$ & 0 \\
$20: 4 n-3$ & $9 \cdot 1$ & 0 \\
$20: 5 n-3$ & $17 \cdot 5$ & 0 \\
$22: 5 n-6$ & $155 \cdot 4$ & 0 \\
$22: 6 n-3$ & $380 \cdot 0$ & 0 \\
\hline
\end{tabular}

excluded if they showed unwillingness to abstain from the consumption of oily fish, fish oil supplements, algal oil supplements, or flaxseed (linseed) oil supplements throughout the course of the study. Subjects received a small financial incentive for their cooperation in the study. The protocol was reviewed and approved by the Research Ethics Committee of King's College London and participants gave informed written consent before commencing the study.

Subjects attended a screening clinic where a small fasting venous blood sample $(17 \mathrm{ml})$ was obtained for measurement of serum lipid profile, blood counts, blood glucose and liver function tests. A urinary pregnancy test was conducted on premenopausal women to confirm non-pregnant status. Habitual dietary intake was assessed before the intervention and during intervention by a $3 \mathrm{~d}$ dietary record which was converted to nutrient intake by computer from food tables based on data using software from CompEat (version 4; Nutrition Systems, Grantham, Lincolnshire, UK).

\section{Measurements}

Seated blood pressure and body weight in minimum indoor clothing were recorded on two consecutive days at baseline and on days 28 and 29 of treatment. Height (to $0 \cdot 1 \mathrm{~cm}$ ) without shoes and weight (to $0.1 \mathrm{~kg}$ ) in minimum indoor clothing were measured using a stadiometer and beam balance respectively. Seated blood pressure was measured using an automated sphygmomanometer (Omron HEM 705-CP; Omron Healthcare Inc., Bannockburn, IL, USA); three blood pressure measurements were made at intervals of $1 \mathrm{~min}$ and the average taken. The subjects recorded in diaries any signs of illness, medication used, menstrual phase and any deviations from the protocol. Subjects were asked to return any unused capsules and a capsule count was made to check compliance. At the end of the study, subjects were requested to complete a questionnaire about their appreciation of the supplements and about any experienced side effects (headache, stomach complaints, nausea, bloated feeling, flatulence, diarrhoea, constipation, itching, eruptions or rashes, fatigue, dizziness, and heavy menstrual period).

The study was conducted double blind except for the measurements of factor VII antigen (FVIIag) and factor VII activated (FVIIa) concentrations and LDL size which were conducted post hoc, but the technicians who made the measurements were blind to the allocation of treatment.

\section{Blood sampling}

Venous blood samples were obtained using the vacutainer technique (Becton Dickinson Vacutainer Systems, Plymouth, Devon, UK) following an overnight fast on two occasions at baseline and on days 28 and 29 of treatment. Blood for serum lipids, creatine kinase, troponin-I and liver function tests was collected in a vacutainer containing no anti-coagulant (vacutainer 17490), serum separated by centrifuging at $1500 \mathrm{~g}$ for $15 \mathrm{~min}$ and kept at $4^{\circ} \mathrm{C}$ until analysed within $3 \mathrm{~d}$. For full blood counts, blood was collected into $2 \mathrm{ml}$ potassium EDTA vacutainer tubes (vacutainer 368047) and kept at room temperature until counted on the same day. Blood for fatty acid and fat-soluble vitamin analyses was collected into an EDTA tube (vacutainer 17644 ), chilled to $4^{\circ} \mathrm{C}$, centrifuged at $1500 \mathrm{~g}$ for $15 \mathrm{~min}$, and samples of plasma were frozen at $-80^{\circ} \mathrm{C}$ until analysed. Packed erythrocytes were washed three times with 5 volumes of icecold saline ( $8.9 \mathrm{NaCl} / \mathrm{l})$ containing $40 \mathrm{mg}$ EDTA/l) and lipid extracts were prepared using isopropanol-chloroform (11:7, v/v) 
containing $50 \mathrm{mg}$ butylated hydroxytoluene/l within $2 \mathrm{~d}$ of blood collection. The lipid extracts were stored at $-20^{\circ} \mathrm{C}$ until analysed. Blood $(4.5 \mathrm{ml})$ for determination of fibrinogen, von Willebrand factor antigen, PAI-1 activity and FVII assays was collected into a vacutainer containing $0.5 \mathrm{ml} 0 \cdot 105 \mathrm{M}$-trisodium citrate (vacutainer 367691) and kept at room temperature until completion of centrifugation ( $1500 \mathrm{~g}$ for $15 \mathrm{~min}$ ). Plasma was carefully separated avoiding contamination with platelets within $2 \mathrm{~h}$ of blood collection and $0.25 \mathrm{ml}$ samples were snap-frozen in liquid $\mathrm{N}_{2}$ and stored at $-80^{\circ} \mathrm{C}$ until analysed.

\section{Analytical methods}

Total cholesterol, HDL and TAG concentrations were measured using fully enzymic procedures with reagents from Wako (Neuss, Germany) and assays were conducted on a Technicon DAX48 automated chemistry analyser (Bayer Diagnostics, Newbury, Berkshire, UK). LDL-cholesterol concentration was calculated using the Friedewald formula (Friedewald et al. 1972). LDL size was determined on pre-stained plasma on an iodixanol gradient using a Beckman NVT 65 near-vertical rotor (Davies et al. 2003). Erythrocyte fatty acid composition and plasma fatty acids were determined by capillary GLC (Sanders et al. 1997) and plasma retinol, tocopherol and carotenoid concentrations were determined by HPLC (Thurnham et al. 1988). Liver function tests, troponin-I, plasma glucose concentrations and serum creatine kinase activity were determined using standard procedures at King's College Hospital (Clinical Pathology Accreditation UK Ltd, reference 0396; Sheffield, UK). Sensitive C-reactive protein (CRP) was determined on a COBAS analyser using reagents supplied by Wako Chemicals (ultrasensitive Creactive protein method). Full blood counts were conducted at King's College Hospital on a Sysmex counter (Sysmex UK Ltd, Milton Keynes, UK). Plasma fibrinogen and von Willebrand factor antigen concentration, FVIIc activity, FVIIag and FVIIa concentrations and PAI-1 activity were determined by the Medical Research Council coagulation laboratory at the Wolfson Institute of Preventive Medicine as previously described (Sanders et al. 2001).

\section{Statistical analysis}

Sample-size estimates were based on thirty-two subjects to give power to detect a $0.7 \mathrm{SD}$ unit change in mean values with $80 \%$ power for $P<0 \cdot 05$. In order to increase the statistical power for the lipid assays to $0.5 \mathrm{SD}$ units, analyses were made on blood samples collected on two consecutive days at baseline and at the end of each treatment. Data for serum TAG, CRP and fibrinogen were log-transformed before statistical analysis. Data were analysed by analysis of covariance using the baseline value as a covariate using SPSS/PC version 10 (SPSS Inc., Chicago, IL, USA) with adjustments for age, BMI and sex. Sex $\times$ treatment interactions were tested in order to determine whether there were differences in response between sexes. Where appropriate, comparisons with baseline values were made using a paired $t$ test.

\section{Results}

A total of eighty subjects were recruited but one male subject withdrew from the study for personal reasons (their details are shown in Table 2); there were no statistically significant differences between groups at baseline. The supplements were well tolerated and no significant side effects were reported. The dietary intakes of the seventy-nine subjects who completed the study are shown in Table 3 and did not change on treatment except for the contribution made by the supplement. Liver function tests, haematology, plasma glucose, retinol and carotenoid concentrations, creatine kinase and troponin-I activities (markers of muscle damage and cardiac muscle damage respectively) were not affected by treatment (data not shown).

Table 4 shows the plasma concentrations of stearic acid (18:0), arachidonic acid $(20: 4 n-6)$, EPA $(20: 5 n-3)$, adrenic acid (22:4n-6), DPA $n-6(22: 5 n-6)$ and DHA $(22: 6 n-3)$ were significantly greater and that of palmitoleic acid $(16: 1)$ was lower following the DHA treatment compared with the placebo. Table 5 shows the changes in erythrocyte lipids where the proportions of DHA $(22: 6 n-3)$, EPA $(20: 5 n-3)$ and DPA $(22: 5 n-6)$ increased and those of linoleic acid $(18: 2 n-6)$, dihomo- $\gamma$-linolenic acid $(20: 3 n-6)$ and DPA $n-3(22: 5 n-3)$ fell. Plasma concentrations of $\alpha$-tocopherol adjusted for plasma

Table 2. Details of the study subjects at screening*

(Mean values and standard deviations)

\begin{tabular}{|c|c|c|c|c|c|c|c|c|}
\hline & \multicolumn{4}{|c|}{ Women } & \multicolumn{4}{|c|}{ Men } \\
\hline & \multicolumn{2}{|c|}{ DHA-S (n 20) } & \multicolumn{2}{|c|}{ Placebo (n 20) } & \multicolumn{2}{|c|}{ DHA-S (n 20) } & \multicolumn{2}{|c|}{ Placebo ( $n$ 19) } \\
\hline Age (years) & 31.6 & $13 \cdot 2$ & $35 \cdot 2$ & 14.5 & $29 \cdot 8$ & $11 \cdot 5$ & 33.4 & $14 \cdot 7$ \\
\hline Height $(\mathrm{cm})$ & 164.9 & $5 \cdot 6$ & $166 \cdot 1$ & 6.9 & $178 \cdot 7$ & $6 \cdot 2$ & $180 \cdot 1$ & $8 \cdot 6$ \\
\hline Weight (kg) & $63 \cdot 8$ & 9.4 & 63.8 & $10 \cdot 2$ & $78 \cdot 1$ & $10 \cdot 1$ & $79 \cdot 0$ & $14 \cdot 8$ \\
\hline Alcohol intake (10 ml units/week) & 7.9 & $5 \cdot 7$ & $5 \cdot 6$ & $5 \cdot 9$ & $10 \cdot 7$ & $9 \cdot 5$ & $10 \cdot 3$ & 9.5 \\
\hline Fasting plasma glucose (mmol/l) & 4.46 & 0.40 & 4.57 & 0.53 & 4.61 & 0.54 & $4 \cdot 74$ & 0.37 \\
\hline Serum cholesterol $(\mathrm{mmol} / \mathrm{l})$ & 4.59 & 0.97 & $4 \cdot 73$ & 0.86 & 4.42 & 0.8 & 3.99 & 0.80 \\
\hline Serum HDL-cholesterol (mmol/l) & $1 \cdot 75$ & 0.47 & 1.69 & 0.46 & $1 \cdot 3$ & 0.3 & 1.4 & 0.2 \\
\hline Serum triacylglycerols $(\mathrm{mmol} / \mathrm{l})$ & 1.07 & 0.57 & 1.04 & 0.61 & 1.4 & 0.9 & $1 \cdot 2$ & 0.6 \\
\hline Systolic blood pressure $(\mathrm{mmHg})$ & $116 \cdot 6$ & $12 \cdot 5$ & $117 \cdot 0$ & $13 \cdot 1$ & $126 \cdot 7$ & $11 \cdot 8$ & $125 \cdot 4$ & $11 \cdot 1$ \\
\hline Diastolic blood pressure $(\mathrm{mmHg})$ & $67 \cdot 8$ & $9 \cdot 4$ & 72.6 & $9 \cdot 2$ & $75 \cdot 0$ & $7 \cdot 7$ & $75 \cdot 3$ & $9 \cdot 7$ \\
\hline
\end{tabular}

*There were no statistically significant differences between treatment groups (two-sample $t$ test). 
Table 3. Nutrient intakes and body weights in subjects before and during treatment with docosahexaenoic acid-rich oil derived from Schizochytrium $s p$. (DHA-S) or placebo*

(Mean values and standard deviations)

\begin{tabular}{|c|c|c|c|c|c|c|c|c|}
\hline & \multicolumn{4}{|c|}{ DHA-S ( $n$ 39) } & \multicolumn{4}{|c|}{ Placebo ( $n$ 39) } \\
\hline & \multicolumn{2}{|c|}{ Baseline } & \multicolumn{2}{|c|}{ End of study } & \multicolumn{2}{|c|}{ Baseline } & \multicolumn{2}{|c|}{ End of study } \\
\hline & Mean & SD & Mean & SD & Mean & SD & Mean & SD \\
\hline Energy (MJ/d) & $7 \cdot 4$ & 2.5 & 7.8 & $2 \cdot 5$ & $8 \cdot 0$ & 2.5 & 7.5 & $2 \cdot 00$ \\
\hline Protein (\% energy) & $14 \cdot 4$ & 2.5 & $15 \cdot 3$ & $4 \cdot 6$ & 14.9 & $2 \cdot 8$ & $14 \cdot 4$ & $2 \cdot 4$ \\
\hline Fat (\% energy) & $32 \cdot 2$ & $3 \cdot 4$ & 34.4 & $8 \cdot 2$ & $35 \cdot 0$ & $7 \cdot 6$ & $34 \cdot 8$ & $6 \cdot 7$ \\
\hline Saturated fatty acids (\% energy) & $11 \cdot 2$ & $3 \cdot 4$ & $11 \cdot 3$ & 4.5 & $12 \cdot 6$ & 4 & $12 \cdot 4$ & $4 \cdot 4$ \\
\hline PUFA (\% energy) & 4.7 & $2 \cdot 1$ & 5.4 & $2 \cdot 1$ & 4.9 & $2 \cdot 2$ & $5 \cdot 3$ & $2 \cdot 2$ \\
\hline Cholesterol $(\mathrm{mg} / \mathrm{d})$ & 210 & 90 & 195 & 109 & 227 & 139 & 217 & 127 \\
\hline Carbohydrate (\% energy) & $49 \cdot 2$ & $7 \cdot 6$ & $46 \cdot 1$ & 8.4 & 44.9 & 8.5 & $46 \cdot 1$ & $7 \cdot 6$ \\
\hline Sugar (\% energy) & $19 \cdot 8$ & 7.5 & $18 \cdot 7$ & $8 \cdot 2$ & $17 \cdot 8$ & $7 \cdot 7$ & $19 \cdot 3$ & 7.5 \\
\hline Starch (\% energy) & $25 \cdot 0$ & $6 \cdot 2$ & $23 \cdot 3$ & $7 \cdot 7$ & $23 \cdot 2$ & $6 \cdot 2$ & $22 \cdot 8$ & $6 \cdot 3$ \\
\hline $\operatorname{NSP}(g / d)$ & $16 \cdot 1$ & 6.7 & $13 \cdot 1$ & $5 \cdot 7$ & $15 \cdot 7$ & $6 \cdot 3$ & $11 \cdot 6$ & $4 \cdot 1$ \\
\hline Body weight (kg) & $70 \cdot 9$ & $12 \cdot 0$ & 71.6 & $2 \cdot 1$ & $71 \cdot 2$ & $14 \cdot 6$ & 71.6 & $14 \cdot 7$ \\
\hline
\end{tabular}

* There were no statistically significant differences between treatments and no statistically significant sex $\times$ treatment interactions (analysis of covariance).

cholesterol concentrations were not affected by the treatment: $5 \cdot 8$ (SD $1 \cdot 1 ; n$ 38) v. 5.8 (SD 1.2) $\mu \mathrm{mol} / \mathrm{mmol}$ cholesterol before and after DHA treatment compared with 6.0 (SD 1.1) v. 5.9 (SD 0.9) $\mu \mathrm{mol} / \mathrm{mmol}$ cholesterol on placebo. Table 6 shows the results for serum lipids; there were no sex $\times$ treatment interactions so the data for both sexes have been combined. Total serum cholesterol, LDL- and HDL-cholesterol concentrations increased following the DHA-S supplement (all $P \leq 0 \cdot 001$ ). Serum TAG concentrations fell slightly following DHA-S compared with baseline (paired $t$ test; $P=0.002$ ), but not on placebo; the difference between treatments did not quite achieve statistical significance. Out of the seventy-seven on whom LDL size was determined, only ten subjects (three in the placebo group and seven in the DHA-treated group) showed a predominance of heavy LDL. There were no significant changes in LDL size between treatments. Table 7 shows the results for blood pressure, CRP and haemostatic variables. There was a trend for systolic blood pressure to fall on the DHA-S treatment (paired t test compared with baseline; $P=0.0004)$, but this did not achieve statistical significance $(P=0 \cdot 103)$ when compared with the placebo. Compared with the baseline, FVIIc increased following the DHA-S treatment compared with the placebo $(P=0.006)$. At baseline, FVIIc was correlated with serum HDL- $(r$ 0.366; $P=0.001)$ and total cholesterol concentrations ( $r$ 0.289; $P=0 \cdot 01)$. However, post hoc analyses of FVIIag or FVIIa concentrations failed to reveal any differences between treatments.

\section{Discussion}

The purpose of the present study was to evaluate the effect of an intake of $1.5 \mathrm{~g}$ DHA/d provided as DHA-S on cardiovascular risk factors in healthy men and women. DHA-S was well tolerated by the subjects and did not result in any self-reported adverse effects or any pathological changes in haematology or other biochemical

Table 4. Plasma fatty acid concentrations $(\mathrm{mg} / \mathrm{l})$ in subjects before and after treatment with docosahexaenoic acid-rich oil derived from Schizochytrium sp. (DHA-S) or placebo

(Mean values, standard deviations and $95 \%$ confidence intervals)

\begin{tabular}{|c|c|c|c|c|c|c|c|c|c|c|c|}
\hline \multirow[b]{3}{*}{ Fatty acids } & \multicolumn{4}{|c|}{ DHA-S ( $n$ 40) } & \multicolumn{4}{|c|}{ Placebo ( $n$ 39) } & \multirow[b]{3}{*}{$P$ value } & \multirow{2}{*}{\multicolumn{2}{|c|}{ Treatment effect* }} \\
\hline & \multicolumn{2}{|c|}{ Baseline } & \multicolumn{2}{|c|}{ End of study } & \multicolumn{2}{|c|}{ Baseline } & \multicolumn{2}{|c|}{ End of study } & & & \\
\hline & Mean & SD & Mean & SD & Mean & SD & Mean & SD & & Mean & $95 \% \mathrm{Cl}$ \\
\hline $16: 0$ & 689 & 236 & 698 & 231 & 576 & 248 & 571 & 261 & NS & 53 & $-36,142$ \\
\hline $16: 1$ & 21 & 25 & 18 & 21 & 22 & 20 & 25 & 24 & NS & -6 & $-14,1$ \\
\hline $18: 0$ & 217 & 72 & 235 & 74 & 198 & 70 & 198 & 65 & $<0.05$ & 27 & 1,52 \\
\hline $18: 1 n-9$ & 656 & 322 & 541 & 338 & 485 & 322 & 566 & 339 & NS & -122 & $-257,13$ \\
\hline $18: 2 n-6$ & 938 & 282 & 951 & 289 & 839 & 253 & 828 & 222 & NS & 64 & $-130,57$ \\
\hline $18: 3 n-3$ & 23 & 11 & 24 & 14 & 20 & 10 & 22 & 14 & NS & -1 & $-6,5$ \\
\hline $20: 3 n-6$ & 50 & 23 & 44 & 25 & 44 & 16 & 47 & 25 & NS & -7 & $-17,3$ \\
\hline $20: 4 n-6$ & 202 & 60 & 223 & 66 & 181 & 62 & 177 & 60 & $<0.01$ & 31 & 10,53 \\
\hline $20: 5 n-3$ & 31 & 25 & 44 & 24 & 37 & 33 & 30 & 20 & $<0.01$ & 15 & 6,24 \\
\hline $22: 4 n-6$ & 6 & 8 & 17 & 15 & 4 & 5 & 4 & 4 & $<0.0001$ & 13 & 8,18 \\
\hline $22: 5 n-6$ & 13 & 13 & 25 & 17 & 9 & 13 & 11 & 12 & $<0.001$ & 11 & 4,16 \\
\hline $22: 5 n-3$ & 35 & 17 & 42 & 24 & 30 & 18 & 33 & 17 & NS & 7 & $-2,15$ \\
\hline $22: 6 n-3$ & 77 & 36 & 165 & 72 & 76 & 48 & 72 & 39 & $<0.0001$ & 92 & 69,115 \\
\hline
\end{tabular}


Table 5. Erythrocyte phospholipid fatty acid composition (weight \%) in subjects before and after treatment with docosahexaenoic acidrich oil derived from Schizochytrium $s p$. (DHA-S) or placebo

(Mean values, standard deviations and $95 \%$ confidence intervals)

\begin{tabular}{|c|c|c|c|c|c|c|c|c|c|c|c|}
\hline \multirow[b]{3}{*}{ Fatty acids } & \multicolumn{4}{|c|}{ DHA-S ( $n$ 40) } & \multicolumn{4}{|c|}{ Placebo ( $n$ 39) } & \multirow[b]{3}{*}{$P$ value } & \multirow{2}{*}{\multicolumn{2}{|c|}{ Treatment effect* }} \\
\hline & \multicolumn{2}{|c|}{ Baseline } & \multicolumn{2}{|c|}{ End of study } & \multicolumn{2}{|c|}{ Baseline } & \multicolumn{2}{|c|}{ End of study } & & & \\
\hline & Mean & SD & Mean & SD & Mean & SD & Mean & SD & & Mean & $95 \% \mathrm{Cl}$ \\
\hline $16: 0$ & $19 \cdot 9$ & 1.5 & $20 \cdot 2$ & $1 \cdot 1$ & $19 \cdot 7$ & 0.9 & $19 \cdot 6$ & 0.9 & $<0.05$ & 0.5 & $0.1,1 \cdot 0$ \\
\hline $16: 1$ & 0.3 & 0.1 & 0.2 & 0.1 & 0.3 & 0.1 & 0.2 & 0.1 & NS & 0 & $-0 \cdot 1,0$ \\
\hline $18: 0$ & $16 \cdot 4$ & 0.9 & $16 \cdot 4$ & 1.0 & $16 \cdot 6$ & 0.9 & $16 \cdot 3$ & $2 \cdot 9$ & NS & -0.1 & $-0.4,0.2$ \\
\hline $18: 1 n-9$ & $13 \cdot 9$ & 2.5 & $14 \cdot 1$ & 1.4 & $14 \cdot 3$ & $1 \cdot 2$ & $13 \cdot 9$ & $2 \cdot 5$ & NS & -0.2 & $-0.6,0.2$ \\
\hline $18: 2 n-6$ & $11 \cdot 3$ & $1 \cdot 0$ & $10 \cdot 6$ & $1 \cdot 1$ & $11 \cdot 3$ & $1 \cdot 1$ & $11 \cdot 1$ & $2 \cdot 1$ & $<0.001$ & -0.7 & $-1 \cdot 1,-0.4$ \\
\hline $20: 3 n-6$ & 1.8 & 0.4 & 1.7 & 0.4 & 1.8 & 0.3 & 1.8 & 0.4 & $<0.01$ & -0.2 & $-0.3,-0.1$ \\
\hline $20: 4 n-6$ & $16 \cdot 7$ & 1.4 & $15 \cdot 8$ & 1.6 & $16 \cdot 0$ & $1 \cdot 7$ & $15 \cdot 4$ & 3.0 & NS & -0.2 & $-0.7,0.2$ \\
\hline $20: 5 n-3$ & $1 \cdot 1$ & 0.4 & $1 \cdot 3$ & 0.4 & $1 \cdot 3$ & 0.7 & $1 \cdot 2$ & 0.6 & $<0.05$ & 0.2 & $0,0.3$ \\
\hline $22: 4 n-6$ & $3 \cdot 1$ & 0.9 & 2.9 & 0.9 & 2.9 & 0.9 & $2 \cdot 7$ & $1 \cdot 2$ & NS & -0.2 & $-0.4,0$ \\
\hline $22: 5 n-6$ & 0.5 & 0.2 & 0.8 & 0.3 & 0.4 & 0.2 & 0.4 & 0.2 & $<0.0001$ & 0.4 & $0.3,0.5$ \\
\hline $22: 5 n-3$ & $2 \cdot 8$ & 0.8 & $2 \cdot 5$ & 0.8 & $2 \cdot 9$ & 1.0 & $2 \cdot 8$ & $1 \cdot 1$ & $<0.0001$ & -0.4 & $-0.5,-0.3$ \\
\hline $22: 6 n-3$ & $6 \cdot 1$ & $1 \cdot 1$ & $7 \cdot 6$ & 1.5 & 6.5 & 1.7 & $6 \cdot 1$ & $2 \cdot 1$ & $<0.0001$ & $1 \cdot 7$ & $1 \cdot 1,2 \cdot 2$ \\
\hline
\end{tabular}

* Treatment effect was analysed by analysis of covariance using the baseline value as a covariate; there were no statistically significant sex $\times$ treatment interactions.

indices. Although the present study was not specifically designed to compare DHA-S with DHA-derived C. cohnii, we did note a different effect on plasma and erythrocyte lipids compared with our previous studies on C. cohnii and fish oil (Sanders et al. 1981, 1997; Sanders \& Hinds, 1992). With TAG derived from C. cohnii, a dose-response decline in arachidonic acid of $11 \%$ with $0.7 \mathrm{~g}$ DHA/d and $18 \%$ with $2.6 \mathrm{~g}$ of DHA/d was observed (T. A. B. Sanders and H. E. Theobald, unpublished results). In the present study, supplementation with DHA-S did not result in a decline in the proportion of arachidonic acid $(20: 4 n-6)$ in plasma or erythrocyte lipids; indeed there was a significant increase in the plasma concentration. Furthermore, DHA-S resulted in a significant increase in DPA $n-6$ (22:5n-6) in both plasma and erythrocyte lipids, a phenomenon hitherto not previously observed in our laboratory, which has experience of over twenty studies in this area. One likely explanation is that DPAn-6 (22:5n-6) is retroconverted to arachidonic acid in a similar manner in which DHA is retroconverted to EPA (Schlenk et al. 1969).
DHA has been shown to lower plasma TAG in subjects with hypertriacylglycerolaemia using intakes considerably greater than in the present study (Davidson et al. 1997; Mori et al. 2000). The DHA-S supplement increased LDL-cholesterol concentrations by $11 \%$, which is consistent with a previous report in moderately hypercholesterolaemic subjects where an intake of $0.7 \mathrm{~g}$ DHA derived from C. cohnii resulted in a $7 \%$ increase in LDL-cholesterol (Theobald et al. 2004). Some studies with fish oil containing DHA have reported increases in LDLcholesterol or LDL apo B concentrations, particularly in hypertriacylglycerolaemic subjects (Sullivan et al. 1986; Harris, 1997). It has been suggested that LDL particles may be lighter following an increased intake of long-chain $n-3$ fatty acids, particularly in subjects with elevated plasma TAG concentrations and a predominance of dense LDL (Minihane et al. 2000). In order to address this question, measurements of LDL size were undertaken post hoc but we were unable to demonstrate any evidence of a change in the proportion of dense LDL or in the peak

Table 6. Serum lipid concentrations in subjects before and after treatment with docosahexaenoic acid-rich oil derived from Schizochytrium sp. (DHA-S) or placebo

(Mean values, standard deviations and $95 \%$ confidence intervals)

\begin{tabular}{|c|c|c|c|c|c|c|c|c|c|c|c|}
\hline & \multicolumn{4}{|c|}{ DHA-S ( $n$ 40) } & \multicolumn{4}{|c|}{ Placebo ( $n$ 39) } & \multirow{2}{*}{$P$ value } & \multirow{2}{*}{\multicolumn{2}{|c|}{ Treatment effect* }} \\
\hline & \multicolumn{2}{|c|}{ Baseline } & \multicolumn{2}{|c|}{ End of study } & \multicolumn{2}{|c|}{ Baseline } & \multicolumn{2}{|c|}{ End of study } & & & \\
\hline Serum cholesterol (mmol/l) & 4.58 & 0.92 & 4.77 & 0.91 & 4.45 & 1.03 & $4 \cdot 33$ & 0.91 & 0.0005 & 0.35 & $0.19,0.50$ \\
\hline Serum LDL-cholesterol (mmol/l) & 2.52 & 0.91 & $2 \cdot 69$ & 0.96 & $2 \cdot 47$ & 0.79 & $2 \cdot 39$ & 0.72 & 0.0002 & 0.27 & $0.13,0.40$ \\
\hline Serum HDL-cholesterol $(\mathrm{mmol} / \mathrm{l})$ & $1 \cdot 52$ & 0.40 & 1.62 & 0.40 & 1.53 & 0.39 & 1.50 & 0.34 & 0.001 & 0.14 & $0.05,0.21$ \\
\hline LDL-I + II (\%) & $72 \cdot 1$ & $14 \cdot 7$ & $72 \cdot 1$ & $14 \cdot 1$ & $78 \cdot 3$ & $10 \cdot 8$ & $77 \cdot 6$ & $12 \cdot 6$ & 0.846 & -0.3 & $-3 \cdot 6,2 \cdot 9$ \\
\hline LDL-III (\%) & $27 \cdot 9$ & $14 \cdot 7$ & $27 \cdot 9$ & $13 \cdot 1$ & $21 \cdot 6$ & $10 \cdot 8$ & $22 \cdot 4$ & $12 \cdot 6$ & 0.846 & 0.3 & $-2 \cdot 9,3 \cdot 6$ \\
\hline Serum triacylglycerols $(\mathrm{mmol} / \mathrm{l}) \dagger$ & 1.08 & 0.50 & 0.93 & 0.41 & 0.92 & 0.43 & 0.90 & 0.44 & 0.152 & -0.09 & $-0.21,0.03$ \\
\hline
\end{tabular}

*Treatment effect was analysed by analysis of covariance using the baseline value as a covariate with adjustments for age, sex and BMI; there were no statistically significant sex $\times$ treatment interactions.

† Geometric mean. 


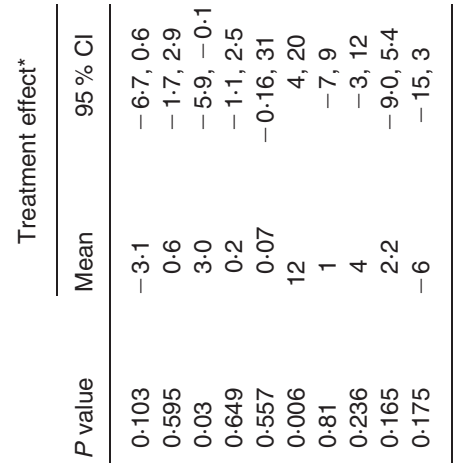

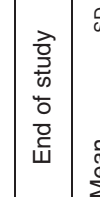

की

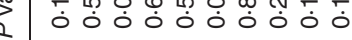

+
+
+

ํㅣㅇํำ

홈

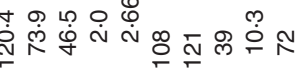

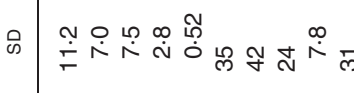

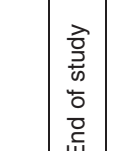

帝

要

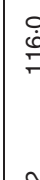

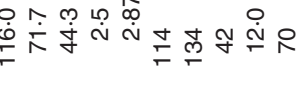

$\stackrel{\sim}{\stackrel{\sim}{\sim}} \stackrel{\infty}{\sim} \dot{+}$

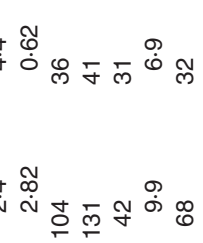

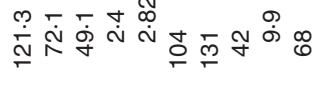

density of LDL. The increase in LDL-cholesterol observed with algal oils rich in DHA appears not to occur in studies using fish oil at equivalent dosages in normolipidaemic subjects (Sanders et al. 1997; Finnegan et al. 2003). This raises the intriguing question of why DHA-S and C. cohnii possess this LDL-raising effect. The amount of saturated fatty acids $(1.2 \mathrm{~g} / \mathrm{d})$ and cholesterol $(32 \mathrm{mg} / \mathrm{d})$ provided by the Schizochytrium $s p$. supplement was low (less than the amounts present in fish oil) and so cannot explain this LDL-cholesterol-raising effect. Further research into the mechanisms involved is warranted.

The results of studies with fish oil have been variable with regard to the effects of long-chain $n$-3 fatty acids on HDL-cholesterol. Harris (1997) concluded that there is a trend for a $3 \%$ increase in HDL-cholesterol concentration in normolipidaemic subjects, which is in agreement with a controlled metabolic feeding study (Sanders et al. 1997) where a significant increase in $\mathrm{HDL}_{2}$ cholesterol concentration but a non-significant $3 \%$ increase in total HDL-cholesterol was demonstrated with diets providing $1.6 \mathrm{~g} \mathrm{DHA} / \mathrm{d}$ and $3.4 \mathrm{~g} \mathrm{EPA} / \mathrm{d}$. The $9 \%$ increase in HDL-cholesterol observed in the present study is much greater than changes previously observed in studies with fish oils. One possible reason for this increase in HDL-cholesterol could be DPA $n-6$ in the Schizochytrium sp. supplement. The observed effects on lipid metabolism might be a consequence of long-chain PUFA, especially DPA $n-6$, acting as a ligand for the liver $\mathrm{X}$ receptor (Yoshikawa et al. 2002) which activates the ABC-A1 transporter (Tontonoz \& Mangelsdorf, 2003) which stimulates reverse cholesterol transport. Although the supplement increased LDL, which is associated with an increased risk of CVD, it also increased HDL-cholesterol, which is associated with a decreased risk. As neither the LDL:HDL ratio nor LDL size changed this would imply that the lipoprotein changes induced by the Schizochytrium sp. supplement have a net neutral effect on cardiovascular risk.

The present study was unable to detect any effect of DHA-S on CRP or fibrinogen concentrations or PAI-1 activity. A striking observation was the $12 \%$ increase in FVIIc on the DHA-S. The increase in FVIIc may be related to the increase in HDL- and LDL-cholesterol concentrations, as FVIIc was correlated with both HDL- and LDL-cholesterol concentrations. In order to ascertain whether the increase in FVIIc was attributable to an increase in zymogen or in FVIIa, we conducted analyses post hoc of FVIIag and FVIIa, but these assays were unable to demonstrate any significant changes in either measure.

A systematic review found a significant blood pressure-lowering effect on long-chain $n$-3 fatty acids (Geleijnse et al. 2002), particularly in subjects with hypertension. In the present study which was conducted in normotensive subjects there was a trend for systolic blood pressure to be $3 \mathrm{mmHg}$ lower in the subjects treated with the Schizochytrium sp. Supplement, but the study lacked sufficient statistically power to detect such a change. Consequently, a much larger study would be needed to test whether intakes of DHA as used in the present study have a blood pressure-lowering effect.

In conclusion, the present study demonstrates that the consumption of oil from Schizochytrium sp. does not result in a reduction in the proportions of arachidonic acid in blood lipids. It raises LDLcholesterol significantly but also HDL-cholesterol concentrations without altering the LDL:HDL ratio or LDL size. Overall, the oil was well tolerated and did not adversely affect cardiovascular risk. 


\section{Acknowledgements}

The authors are grateful to Dr Roy Sherwood for supervising the lipoprotein analyses and clinical chemistry, David Howarth for the haemostatic assays, Robert Gray for the fatty acid and vitamin analyses, and Ian Davies for the measurement of LDL size. The present study was funded by Omega-Tech Inc., which is now part of the Martek Biosciences Corporation. T. A. B. S. was the principal investigator; K. G. recruited the subjects for the study and was responsible for the day-to-day running of the study; B. A. G. developed the method for determining LDL size and contributed to the preparation of the manuscript; G. J. M. contributed to the design, interpretation and preparation of the manuscript. We declare there are no conflicting interests.

\section{References}

Albert CM, Hennekens CH, O'Donnell CJ, Ajani UA, Carey VJ, Willett WC, Ruskin JN \& Manson JE (1998) Fish consumption and risk of sudden cardiac death. JAMA 279, 23-28.

British Nutrition Foundation (1992) Unsaturated Fatty Acids: Nutritional and Physiological Significance: The Report of the British Nutrition Foundation's Task Force. London: Chapman and Hall.

Bucher HC, Hengstler P, Schindler C \& Meier G (2002) n-3 Polyunsaturated fatty acids in coronary heart disease: a meta-analysis of randomized controlled trials. Am J Med 112, 298-304.

Cooper JA, Miller GJ, Bauer KA, Morrissey JH, Meade TW, Howarth DJ, Barzegar S, Mitchell JP \& Rosenberg RD (2000) Comparison of novel hemostatic factors and conventional risk factors for prediction of coronary heart disease. Circulation 102, 2816-2822.

Davidson MH, Maki KC, Kalkowski J, Schaefer EJ, Torri SA \& Drennan KB (1997) Effects of docosahexaenoic acid on serum lipoproteins in patients with combined hyperlipidemia: a randomized, double-blind, placebo-controlled trial. J Am Coll Nutr 16, 236-243.

Davies IG, Graham JM \& Griffin BA (2003) Rapid separation of LDL subclasses by iodixanol gradient ultracentrifugation. Clin Chem 49, $1865-1872$

Finnegan YE, Minihane AM, Leigh-Firbank EC, Kew S, Meijer GW, Muggli R, Calder PC \& Williams CM (2003) Plant- and marine-derived n-3 polyunsaturated fatty acids have differential effects on fasting and postprandial blood lipid concentrations and on the susceptibility of LDL to oxidative modification in moderately hyperlipidemic subjects. Am J Clin Nutr 77, 783-795.

Friedewald WT, Levi RI \& Fredrickson DS (1972) Estimation of the concentration of low density lipoprotein cholesterol in plasma without use of the ultracentrifuge. Clin Chem 18, 499-502.

Geleijnse JM, Giltay EJ, Grobbee DE, Donders AR \& Kok FJ (2002) Blood pressure response to fish oil supplementation: metaregression analysis of randomized trials. J Hypertens 20, 1493-1499.

Harris WS (1997) n-3 Fatty acids and serum lipoproteins: human studies. Am J Clin Nutr 65, Suppl., S1645-S1654.

Hu FB, Bronner L, Willett WC, Stampfer MJ, Rexrode KM, Albert CM, Hunter D \& Manson JE (2002) Fish and omega-3 fatty acid intake and risk of coronary heart disease in women. JAMA 287, 1815-1821.

Makrides M, Neumann MA, Jeffrey B, Lien EL \& Gibson RA (2000) A randomized trial of different ratios of linoleic to alpha-linolenic acid in the diet of term infants: effects on visual function and growth. Am J Clin Nutr 71, 120-129.

Marchioli R, Barzi F, Bomba E, et al. (2002) Early protection against sudden death by $n-3$ polyunsaturated fatty acids after myocardial infarction: time-course analysis of the results of the Gruppo Italiano per lo Studio della Sopravvivenza nell'Infarto Miocardico (GISSI)-Prevenzione. Circulation 105, 1897-1903.

Meade TW, Ruddock V, Stirling Y, Chakrabarti R \& Miller GJ (1993) Fibrinolytic activity, clotting factors, and long-term incidence of ischaemic heart disease in the Northwick Park Heart Study. Lancet 342, 1076-1079.

Metz JG, Roessler P, Facciotti D, Levering C, Dittrich F, Lassner M, Valentine R, Lardizabal K, Domergue F, Yamada A, Yazawa K, Knauf V \& Browse J (2001) Production of polyunsaturated fatty acids by polyketide synthases in both prokaryotes and eukaryotes. Science 293, 290-293.

Minihane AM, Khan S, Leigh-Firbank EC, Talmud P, Wright JW, Murphy MC, Griffin BA \& Williams CM (2000) ApoE polymorphism and fish oil supplementation in subjects with an atherogenic lipoprotein phenotype. Arterioscler Thromb Vasc Biol 20, 1990-1997.

Mori TA, Burke V, Puddey IB, Watts GF, O'Neal DN, Best JD \& Beilin LJ (2000) Purified eicosapentaenoic and docosahexaenoic acids have differential effects on serum lipids and lipoproteins, LDL particle size, glucose, and insulin in mildly hyperlipidemic men. Am J Clin Nutr 71, 1085-1094.

Mozaffarian D, Longstreth WT Jr, Lemaitre RN, Manolio TA, Kuller LH, Burke GL \& Siscovick DS (2005) Fish consumption and stroke risk in elderly individuals: The Cardiovascular Health Study. Arch Intern Med 165, 200-206.

Ridker PM, Hennekens CH, Buring JE \& Rifai N (2000) C-reactive protein and other markers of inflammation in the prediction of cardiovascular disease in women. $N$ Eng $J$ Med 342, 836-843.

Rosell MS, Lloyd-Wright Z, Appleby PN, Sanders TA, Allen NE \& Key TJ (2005) Long-chain n-3 polyunsaturated fatty acids in plasma in British meat-eating, vegetarian, and vegan men. Am J Clin Nutr $\mathbf{8 2}$, 327-334.

Sanders TA \& Hinds A (1992) The influence of a fish oil high in docosahexaenoic acid on plasma lipoprotein and vitamin $\mathrm{E}$ concentrations and haemostatic function in healthy male volunteers. $B r J$ Nutr $\mathbf{6 8}$, $163-173$.

Sanders TA, Oakley FR, Cooper JA \& Miller GJ (2001) Influence of a stearic acid-rich structured triacylglycerol on postprandial lipemia, factor VII concentrations, and fibrinolytic activity in healthy subjects. Am J Clin Nutr 73, 715-721.

Sanders TA, Oakley FR, Miller GJ, Mitropoulos KA, Crook D \& Oliver MF (1997) Influence of $n$-6 versus $n-3$ polyunsaturated fatty acids in diets low in saturated fatty acids on plasma lipoproteins and hemostatic factors. Arterioscler Thromb Vasc Biol 17, 3449-3460.

Sanders TA, Vickers M \& Haines AP (1981) Effect on blood lipids and haemostasis of a supplement of cod-liver oil, rich in eicosapentaenoic and docosahexaenoic acids, in healthy young men. Clin Sci (Lond) 61, 317-324.

Schlenk H, Sand DM \& Gellerman JL (1969) Retroconversion of docosahexaenoic acid in the rat. Biochim Biophys Acta 187, 201-207.

Sullivan DR, Sanders TA, Trayner IM \& Thompson GR (1986) Paradoxical elevation of LDL apoprotein B levels in hypertriglyceridaemic patients and normal subjects ingesting fish oil. Atherosclerosis 61, 129-134.

Theobald HE, Chowienczyk PJ, Whittall R, Humphries SE \& Sanders TA (2004) LDL cholesterol-raising effect of low-dose docosahexaenoic acid in middle-aged men and women. Am J Clin Nutr 79, $558-563$.

Thompson SG, Kienast J, Pyke SD, Haverkate F \& van de Loo JC (1995) Hemostatic factors and the risk of myocardial infarction or sudden death in patients with angina pectoris. European Concerted Action on Thrombosis and Disabilities Angina Pectoris Study Group. $N$ Engl $J$ Med 332, 635-641.

Thurnham DI, Smith E \& Flora PS (1988) Concurrent liquid-chromatographic assay of retinol, alpha-tocopherol, beta-carotene, alpha-carotene, lycopene, and beta-cryptoxanthin in plasma, with tocopherol acetate as internal standard. Clin Chem 34, 377-381.

Tontonoz P \& Mangelsdorf DJ (2003) Liver X receptor signalling pathways in cardiovascular disease. Mol Endocrinol 17, 985-993.

Yoshikawa T, Shimano H, Yahagi N, et al. (2002) Polyunsaturated fatty acids suppress sterol regulatory element-binding protein $1 \mathrm{c}$ promoter activity by inhibition of liver $\mathrm{X}$ receptor (LXR) binding to LXR response elements. J Biol Chem 277, 1705-1711. 